\title{
Detection Uncertainty Matters for Understanding Atmospheric Rivers
}

\author{
Travis A. O’Brien*† \\ Lawrence Berkeley Lab, Berkeley, CA, USA
}

Ashley E. Payne

University of Michigan, Ann Arbor, MI, USA

Christine A. Shields

National Center for Atmospheric Research, Boulder, CO, USA

Jonathan Rutz

9

10 Division, Salt Lake City, UT, USA

National Weather Service, Western Region Headquarters, Science and Technology Infusion

Swen Brands

MeteoGalicia, Galicia, Spain

Christopher Castellano

CW3E, Scripps Institution of Oceanography, University of California San Diego, La Jolla, CA, $U S A$

Jiayi Chen University of California, Berkeley, Berkeley, CA, USA 


\section{William Cleveland}

Purdue, West Lafayette, IN, USA

CW3E, Scripps Institution of Oceanography, University of California San Diego, La Jolla, CA, USA

\author{
Naomi Goldenson \\ University of California, Los Angeles, Los Angeles, CA \\ Irina Gorodetskaya \\ CESAM, University of Aveiro, Portugal
}

Héctor Inda Díaz

University of California, Davis, Davis, CA, USA

Karthik Kashinath

Lawrence Berkeley National Laboratory, Berkeley, CA, USA

Brian Kawzenuk

CW3E, Scripps Institution of Oceanography, La Jolla, CA, USA

Sol Kim

University of California Berkeley, Berkeley CA USA

Mikhail Krinitskiy

Shirshov Institute of Oceanology, Russian Academy of Sciences. Moscow, Russia 
Juan M. Lora

Yale University, New Haven, CT, USA

Beth McClenny

University of California, Davis, Davis, CA, USA

Allison Michaelis

CW3E, Scripps Institution of Oceanography, La Jolla, CA, USA

John O’Brien

University of California, Santa Cruz, Santa Cruz, CA. USA

Christina M. Patricola

Lawrence Berkeley National Laboratory, Berkeley, CA, USA

Eric J. Shearer

Center for Hydrometeorology and Remote Sensing, University of California, Irvine, Irvine, CA, USA

Wen-Wen Tung

Purdue, West Lafayette, IN, USA

Paul Ullrich 
Kevin Yang

${ }_{67}^{*}$ Corresponding author address: Climate and Ecosystem Sciences Division, Lawrence Berkeley

69 E-mail: obrienta@iu.edu

${ }_{70}{ }^{\dagger}$ Current affiliation: Earth and Atmospheric Sciences, Indiana University, Bloomington, IN 


\section{ABSTRACT}

${ }_{71} \quad$ The $3^{\text {rd }}$ ARTMIP Workshop

72 What: Over 30 participants from multiple universities and research insi-

${ }_{73}$ titutions met to discuss new results from the Atmospheric River Tracking

74 Method Intercomparison Project.

75 Where: Lawrence Berkeley National Lab, Berkeley, CA, USA

${ }_{76}$ When: 16-18 October 2019

77 
Atmospheric rivers (ARs) are increasingly recognized globally as an important weather phenomenon associated with extreme precipitation. There is a substantial body of literature indicating that ARs are responsible for a large fraction of wet-season precipitation on western coasts (Rutz et al. 2019) and that they can cause large changes in snowpack (both positive and negative; Guan et al. 2010; Chen et al. 2019). Individual ARs and collections of ARs can bring large amounts of precipitation that drives floods and other storm-related hazards (Ralph et al. 2006, 2019a). ARs are a significant factor for water and associated water systems in the vicinity of western coasts (Gao et al. 2016; Ralph et al. 2019b). It is increasingly evident that they have major impacts on the energy and water budgets of the cryosphere: including mountains (Chen et al. 2019) and high latitude regions (Gorodetskaya et al. 2014). These research advances hinge on technical advances in tracking ARs in observations, reanalyses, and climate model simulations and on understanding uncertainties associated with different tracking methods. In parallel with the recent increase in research activity around ARs, an increasing number of research groups have developed unique methods for tracking ARs (Shields et al. 2019).

The Atmospheric River Tracking Intercomparison Project (ARTMIP) was created to design a set of experiments that could quantify the uncertainty associated with AR tracking (Shields et al. 2018; Rutz et al. 2019). The concept of a multi-tiered experimental approach, based on tracking ARs across common datasets, resulted from the $1^{\text {st }}$ ARTMIP workshop in 2017. The Tier 1 experiment is focused on tracking ARs in a modern reanalysis (MERRA2). The $2^{\text {nd }}$ ARTMIP workshop (Shields et al. 2019) was oriented around discussion of Tier 1 results and around designing and planning the first set of Tier 2 experiments: the Tier 2 C20C+ experiment and the Tier 2 CMIP5/6 experiment. Both initial Tier 2 experiments are focused on understanding the effects of climate change on AR characteristics, with the $\mathrm{C} 20 \mathrm{C}+$ experiment focusing on a set of high-resolution 
atmosphere-only simulations, and the CMIP5/6 experiment focusing on a multimodel collection of fully-coupled simulations from the Coupled Model Intercomparison Project.

Following the $2^{\text {nd }}$ ARTMIP Workshop, two separate developments motivated the need for developing a large dataset of hand-labeled ARs. Discussions following the $2^{\text {nd }}$ ARTMIP Workshop suggested that differences among AR tracking algorithms might reflect differences in expert opinion about what constitutes the boundary of ARs; resolving this question would require experts to hand-label ARs. Unrelated, but concurrent, advances in Computational Climate Science have demonstrated the utility of modern machine learning methods for tracking weather phenomena (Mudigonda et al. 2017; Muszynski et al. 2019; Kurth et al. 2018). These developments also highlight the need for high-quality data to train machine learning methods: expert-labeled datasets.

Emerging results from the Tier 1 and 2 experiments, along with the recently identified need to develop a high-quality, hand-labeled dataset of ARs, motivated the ARTMIP Committee to convene the 3rd ARTMIP Workshop ${ }^{1}$, held at Lawrence Berkeley Lab on October 16-18, 2019. The meeting included a substantial virtual component, with $25 \%$ of attendees attending virtually; the meeting included several presentations from remote attendees. The 3rd ARTMIP Workshop was organized around:

- presentation of results from recent and ongoing ARTMIP research: Tier 1 and beyond (with a focus on Tier 2);

- working discussion of current and future ARTMIP experiments and papers; and

- solicitation of expert identification of atmospheric rivers and other weather phenomena for machine learning.

\footnotetext{
${ }^{1}$ Funded by the U.S. Department of Energy
} 
Initial Tier 2 results presented at the workshop show that, while most methods agree, qualitative conclusions about the effect of climate change on ARs can depend on tracking algorithm. These results further motivate exploration of the role of AR tracking uncertainty on other aspects of AR science. Specifications and timelines for three new Tier 2 experiments were defined: Tier 2 Reanalysis, Tier 2 High-Latitude, and Tier 2 paleo-ARTMIP. A future Tier 2 experiment was also discussed, and specifications and a timeline will be developed in future ARTMIP interactions (e.g., teleconferences): Tier 2 MPAS-ENSO. Group and breakout discussions during the workshop identified numerous gaps in understanding and associated research priorities. These gaps and research priorities are a key outcome for the ARTMIP workshop. Those interested in more information about the workshop should refer to the full workshop report, which is available at the Department of Energy website.

\section{Key Gaps and Research Priorities}

\section{a. Basic Research on AR Lifecycle}

Gap: The physical drivers of AR genesis, development, and dissipation are not completely understood, and this lack of understanding impedes our ability to constrain the quantitative definition, detection, and tracking of ARs.

Recommendation: There is a need for more basic research on the dynamics and lifecycle of ARs.

There was considerable discussion during the workshop about the need for refining our theoretical understanding of the AR lifecycle: from genesis to dissipation. Some basic questions were identified that, if answered, could help reduce quantitative uncertainty in the definition of ARs: 
1. What causes the genesis of ARs?

2. What controls the frequency of ARs?

3. What controls the duration of ARs?

4. Are ARs always associated with ETCs?

5. Are ARs always associated with some form of baroclinic instability?

Analysis and intercomparison of the dynamics associated with ARs would be a valuable and logical step toward providing answers to some of these questions. Recent work by Zhou et al., which was presented during the workshop, shows that different phases of the MJO initiate equatorial Rossby and Kelvin waves_in a classic Gill response to tropical heating anomalies-that modulate the frequency and location of AR genesis in the Pacific. This analysis addresses questions 1 and 2, and more analyses of this type would help refine our understanding of the formation of ARs.

\section{b. Flavors of ARs}

Gap: Existing tracking methods do not consider that there might be different "flavors" of ARs.

Recommendation: Research is needed to determine whether and how there might be different flavors of ARs (e.g., role of baroclinity, generation mechanisms, etc.), and if so, whether this might lead to different classes of tracking algorithms.

It was also postulated that there might be different "flavors" of ARs, with different generating physical mechanisms controlling their lifecycle; e.g., if some are associated with transient baroclinic instabilities and others are associated with quasi-stationary geopotential height gradients. 
Relatedly, there was also discussion about the utility of analyzing the dynamics (e.g., baroclinicity) associated with ARs across different algorithms. This could provide insight into the underlying dynamical processes that influence the evolution of ARs at various stages of their life cycles.

Prevailing tracking methods have not considered this posibitility. Such methods might require an ability to distinguish among ARs with different physical characteristics, such as tropical moisture filaments, ARs that originate from extra-tropical cyclones, those encompassing uplifting motions versus not, ARs embedded in steering flow, etc. This is a critical step to enable further understanding, accurate identification, and improved forecasting of ARs and associated physical systems. Ideally, "flavored" AR tracking methods could incorporate connections to surface precipitation, interactions with synoptic-scale baroclinicity, and interactions with other phenomena such as tropical cyclones and jet streams.

\section{c. Classes of AR Algorithm}

Gap: ARTMIP has documented different classes of AR detection algorithm, which partially explains the spread in AR detection results.

Recommendation: Objective, and physics-informed, clustering approaches could help establish a quantitative vocabulary for explaining differences among AR detection algorithms.

The range of features detected by algorithms in existing Tier 1 and 2 datasets is an immediate and ongoing source of uncertainty that has provided challenges for those analyzing ARTMIP output. Aside from relative vs. absolute methods, there is no a priori way-at least that the ARTMIP community has so far identified - to group AR detection methods in a way that helps make sense of the broad range of AR characteristics observed across algorithms. 
Despite the focus of the discussion on existing uncertainties in AR detection techniques and impacts on AR science, the group found a cause for cautious optimism: analogous to different physics parameterizations in climate models, different AR algorithms were developed with different goals in mind, and thus may each have distinct applications. This suggests that there exists a logical approach to group and categorize existing AR algorithms to facilitate understanding of how and why AR characteristics and metrics differ among algorithms. The group also discussed the possibility of using statistical methods, such as K-means clustering, to objectively categorize AR detection algorithms. If there are different AR flavors, there is the possibility that different detection methods tend to preferentially identify different AR flavors; objective clustering methods could help clarify this.

\section{d. Leveraging 3D Structure}

Gap: Most current AR detection algorithms are primarily based on 2D features, which is partly due to computational considerations and data availability, but ARs have distinct 3D structure.

Recommendation: Research groups with expertise in, and access to, high performance computing resources should explore detection approaches that leverage the 3D structure of ARs.

The group identified several gaps that may limit the ability of current AR tracking results to improve our understanding and prediction of AR physics and impacts. First, current detection algorithms are all based on two-dimensional horizontal patterns. This choice is partly influenced by the computational resources generally available and by data limitations/availability (e.g., most satellite datasets are 2D). In reality, ARs have complicated three-dimensional structures in nature. The physical features of extra-tropical cyclones likely make simple thresholding methods unfeasible. However, applying detection or tracking algorithms to large, volumetric data is computa- 
tionally highly complex and requires substantial resources (e.g., memory) that make such work impractical for many. Research groups with sufficient computing resources could advance AR science by developing algorithms that consider the three-dimensional nature of ARs.

\section{e. Common Software Infrastructure}

Gap: There are a growing number of different AR detection codes reflecting a diversity of quantitative AR definitions. Software differences make the systematic comparison of these definitions difficult.

Recommendation: Develop open-source computational frameworks to facilitate the implementation of new and existing AR detection methods.

Common open-source computational approaches will help broaden and speed up AR-related research. The community can benefit from some open-source codes that make efficient AR tracking for operational tasks or exploratory studies. In addition, open-source codes showing discretization schemes for calculating terms and equations used for AR identification can help ensure consistency across all related physics-driven data analysis studies at the numerical level. The RGMA-funded Toolkit for Extreme Climate Analysis (TECA) ${ }^{2}$ may prove to be a useful starting point for developing an open-source ARTMIP framework, as it is designed to facilitate the development of modular data processing pipelines on high-performance computing systems.

\section{f. Expert-Labeled AR Dataset}

Gap: It is not clear whether differences among expert opinions about AR boundaries are as large as differences among AR detection algorithms.

\footnotetext{
${ }^{2}$ https://github.com/LBL-EESA/TECA/
} 
Gap: Existing machine learning methods for detecting ARs are based on heuristic algorithms.

Recommendation: Future AR research, especially research using machine learning, should leverage results from the ARTMIP ClimateNet campaign.

A unique component of the 3rd ARTMIP Workshop, relative to previous ARTMIP workshops and to other discipline-focused workshops, is the inclusion of a workshop session devoted to having experts hand-identify ARs. The purpose of the session was twofold: (1) to assess the extent to which differences among algorithms might reflect differences in opinion about what ARs are, and (2) to develop a dataset that can form the basis for machine-learning-based AR detectors.

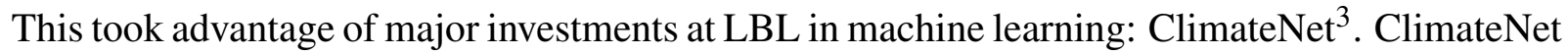
was developed at LBL/NERSC to facilitate the collection of hand-labeled weather datasets. This component of the workshop was substantial: half of a day, out of a 2.5-day workshop, was devoted to this effort. This effort included over 15 workshop participants who labeled 660 time slices of data during the session (Figure 1).

\section{Conclusions}

The enthusiasm for ARTMIP was evident during the workshop, especially when discussing potential future areas of exploration (e.g., new Tier 2 experiments). To this end, plans were made to expand the ARTMIP timeline to include two new Tier 2 subtopics, e.g. Reanalysis sensitivity and Paleoclimate. ARTMIP will continue to provide the community with AR catalogues across all subtopics with the aim of facilitating scientific discourse and forwarding our understanding of atmospheric rivers. We will accomplish this by continuing our activities (Master ARTMIP

\footnotetext{
${ }^{3}$ https://www.nersc.gov/research-and-development/data-analytics/big-data-center/climatenet/
} 
Timeline), contributing to the body of scientific literature, and participating in scientific meetings with a short-term goal of proposing sessions at IARC 2020 in Chile and relevant society meetings.

Acknowledgments. Funding for the 3rd ARTMIP Workshop was provided by the LBNL CASCADE SFA, which is supported by the U.S. Department of Energy, Office of Science, Office of Biological and Environmental Research, Climate and Environmental Sciences Division, Regional \& Global Climate Modeling Program, under Award Number DE-AC02-05CH11231 and used resources of the National Energy Research Scientific Computing Center (NERSC), which is a DOE Office of Science User Facility. Contribution of the Shirshov Institute of Oceanology was supported by the Russian Science Foundation grant 17-77-20112.

\section{References}

Chen, X., L. R. Leung, M. Wigmosta, and M. Richmond, 2019: Impact of atmospheric rivers on surface hydrological processes in western u.s. watersheds. Journal of Geophysical Research: Atmospheres, 124 (16), 8896-8916, doi:10.1029/2019JD030468, URL https://agupubs. onlinelibrary.wiley.com/doi/abs/10.1029/2019JD030468.

Gao, Y., J. Lu, and L. R. Leung, 2016: Uncertainties in projecting future changes in atmospheric rivers and their impacts on heavy precipitation over europe. Journal of Climate, 29 (18), 6711-6726, doi:10.1175/JCLI-D-16-0088.1, URL https://doi.org/10.1175/ jcli-d-16-0088.1,http://journals.ametsoc.org/doi/10.1175/JCLI-D-16-0088.1.

Gorodetskaya, I. V., M. Tsukernik, K. Claes, M. F. Ralph, W. D. Neff, and N. P. M. Van Lipzig, 2014: The role of atmospheric rivers in anomalous snow accumulation in east antarctica. Geophysical Research Letters, 41 (17), 6199-6206, doi:10.1002/2014GL060881, URL http: //doi.wiley.com/10.1002/2014GL060881. 
Guan, B., N. P. Molotch, D. E. Waliser, E. J. Fetzer, and P. J. Neiman, 2010: Extreme snowfall events linked to atmospheric rivers and surface air temperature via satellite measurements. Geophysical Research Letters, 37 (20), n/a-n/a, doi:10.1029/2010GL044696, URL https://doi.org/10.1029/2010GL044696.http://doi.wiley.com/10.1029/2010GL044696.

Kurth, T., and Coauthors, 2018: Exascale Deep Learning for Climate Analytics. Proceedings of the International Conference for High Performance Computing, Networking, Storage, and Analysis, IEEE Press, Piscataway, NJ, USA, 51:1—-51:12, SC '18, URL http://dl.acm.org/citation.cfm? id $=3291656.3291724$.

Mudigonda, M., and Coauthors, 2017: Segmenting and Tracking Extreme Climate Events using Neural Networks. 31 st Conference on Neural Information Processing System, Long Beach, CA, USA, 1-5, Deep Learning for Physical Climate, URL https://dl4physicalsciences.github.io/files/ nips_dlps_2017_20.pdf.

Muszynski, G., K. Kashinath, V. Kurlin, and M. Wehner, 2019: Topological data analysis and machine learning for recognizing atmospheric river patterns in large climate datasets. Geoscientific Model Development, 12 (2), 613-628, doi:10.5194/gmd-12-613-2019, URL https: //doi.org/10.5194/gmd-12-613-2019,https://www.geosci-model-dev.net/12/613/2019/.

Ralph, F. M., P. J. Neiman, G. A. Wick, S. I. Gutman, M. D. Dettinger, D. R. Cayan, and A. B. White, 2006: Flooding on california's russian river: Role of atmospheric rivers. Geophysical Research Letters, 33 (13), L13 801, doi:10.1029/2006GL026689, URL https://doi.org/10.1029/ 2006GL026689.http://doi.wiley.com/10.1029/2006GL026689.

Ralph, F. M., J. J. Rutz, J. M. Cordeira, M. Dettinger, M. Anderson, D. Reynolds, L. J. Schick, and C. Smallcomb, 2019a: A scale to characterize the strength and impacts 
of atmospheric rivers. Bulletin of the American Meteorological Society, 100 (2), 269289, doi:10.1175/BAMS-D-18-0023.1, URL https://doi.org/10.1175/BAMS-D-18-0023.1http: //journals.ametsoc.org/doi/10.1175/BAMS-D-18-0023.1.

Ralph, F. M., and Coauthors, 2019b: ARTMIP-early start comparison of atmospheric river detection tools: how many atmospheric rivers hit northern california's russian river watershed? Climate Dynamics, 52 (7), 4973-4994, doi:10.1007/s00382-018-4427-5, URL https: //doi.org/10.1007/s00382-018-4427-5.,http://link.springer.com/10.1007/s00382-018-4427-5.

Rutz, J. J., and Coauthors, 2019: The atmospheric river tracking method intercomparison project (ARTMIP): Quantifying uncertainties in atmospheric river climatology. Journal Of Geophysical Research-Atmospheres, doi:10.1029/2019JD030936.

Shields, C. A., J. J. Rutz, L. R. Leung, F. M. Ralph, M. Wehner, T. O’Brien, and R. Pierce, 2019: Defining uncertainties through comparison of atmospheric river tracking methods. Bulletin of the American Meteorological Society, 100 (2), ES93-ES96, doi:10.1175/BAMS-D-18-0200. 1, URL https://doi.org/10.1175/BAMS-D-18-0200.1http://journals.ametsoc.org/doi/10.1175/ BAMS-D-18-0200.1.

Shields, C. A., and Coauthors, 2018: Atmospheric river tracking method intercomparison project (ARTMIP): project goals and experimental design. Geoscientific Model Development, 11 (6), 2455-2474, doi:10.5194/gmd-11-2455-2018, URL https://doi.org/10.5194/ gmd-11-2455-2018,https://www.geosci-model-dev.net/11/2455/2018/. 
LIST OF FIGURES

Fig. 1. Comparison of expert AR identifications from 06 September 2009 of a 25 km CAM5 Atmospheric Model Interocomparison Project simulation. The background field shows integrated water vapor, and the green contours show outlines of ARs identified by 15 ARTMIP participants. 


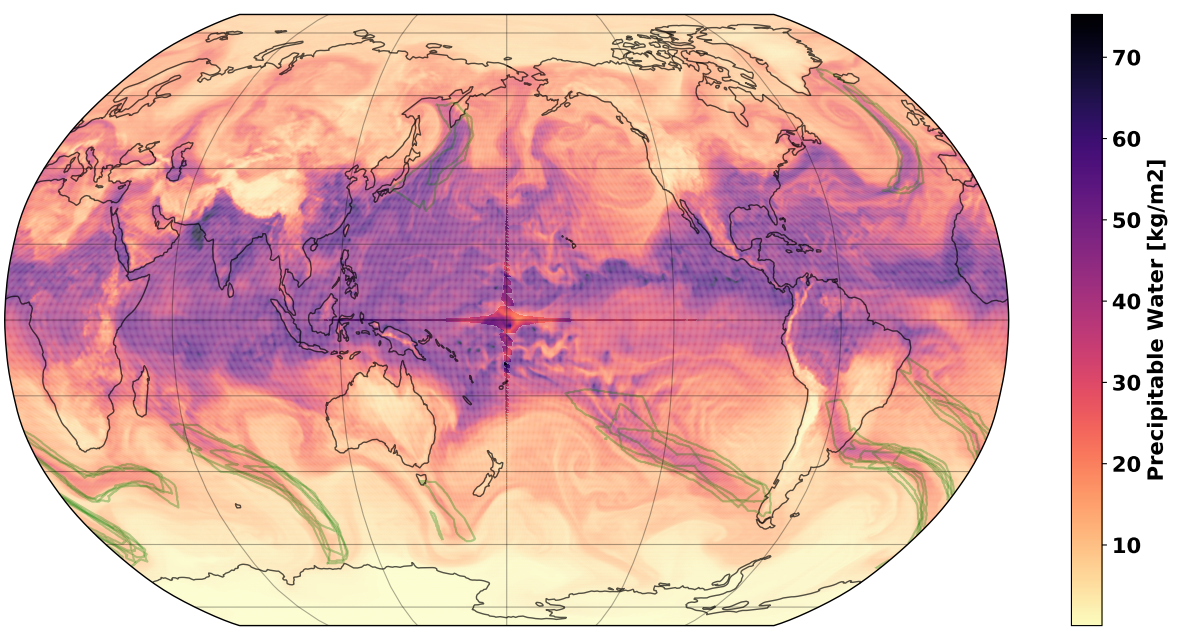

FIG. 1. Comparison of expert AR identifications from 06 September 2009 of a 25 km CAM5 Atmospheric Model Interocomparison Project simulation. The background field shows integrated water vapor, and the green contours show outlines of ARs identified by 15 ARTMIP participants. 